\title{
Blue Stragglers: Spectra of Globular Clusters
}

\author{
A. J. Cenarro ${ }^{1,2}$, J. L. Cervantes ${ }^{1}$, M. A. Beasley ${ }^{1}$, A. Marin-Franch ${ }^{1}$ \\ and A. Vazdekis ${ }^{1}$ \\ ${ }^{1}$ Instituto de Astrofísica de Canarias, \\ Vía Láctea s/n, E-38200 La Laguna, Tenerife, Spain \\ ${ }^{2}$ Centro de Estudios de Física del Cosmos de Aragón, \\ C/ General Pizarro 1, E-44001 Teruel, Spain \\ email: cenarro@cefca.es
}

\begin{abstract}
The integrated Balmer lines of unresolved stellar systems have been widely used as age indicators, since they are sensitive to the temperature of the main sequence turn-off. However, the existence of "non-canonical" stellar stages such as hot horizontal branch stars and blue straggler stars (BSSs) can lead to underestimations of the true stellar population ages. Using an optimized $\mathrm{H} \beta$ index in conjunction with HST/WFPC2 color-magnitude diagrams (CMDs), we find that Galactic globular clusters of similar metallicity exhibit a large scatter in their $\mathrm{H} \beta$ strengths, which does not correlate with their CMD-derived ages. Instead, we demonstrate that the specific frequency of BSSs is responsible for the observed $\mathrm{H} \beta$ scatter at intermediate-to-high metallicity, in the sense that, at fixed metallicity, higher BSS ratios lead to larger integrated $\mathrm{H} \beta$ strengths. Therefore, the specific frequency of BSSs sets a fundamental limit on the accuracy for which integrated spectroscopic ages can be determined for globular clusters and, probably, other stellar systems like galaxies. The observational implications of this result are discussed.
\end{abstract}

Keywords. blue stragglers - globular clusters: general — galaxies: star clusters — galaxies: stellar content

\section{Introduction}

It is well known that the integrated Balmer lines of unresolved stellar populations are very sensitive to the effective temperature $\left(T_{\text {eff }}\right)$ of the main sequence turn-off (MSTO), which in turn is directly related to the population age. Therefore, a common technique for estimating the ages of unresolved stellar systems is to measure both Balmer and metal lines from their integrated spectra and compare them to simple stellar population (SSP) models. However, the presence of bluer horizontal branch stars (HBs) and blue straggler stars (BSSs) in unresolved stellar populations may lead to important underestimations of the spectroscopic ages, as both blue HB stars and BSSs with $T_{\text {eff }}>6500 \mathrm{~K}$ show prominent Balmer lines in their spectra which can mimic the presence of younger stellar populations (e.g. Rose 1985, Lee et al. 2000, Schiavon et al. 2004; Trager et al. 2005; Cenarro et al. 2007b).

Recently, Cervantes \& Vazdekis (2008) defined an optimized line-strength index for $\mathrm{H} \beta$, called $\mathrm{H} \beta_{\mathrm{o}}$, that minimizes the metallicity dependence of the Balmer line in favor of its age sensitivity. Interestingly, the integrated $\mathrm{H} \beta_{\mathrm{o}}$ values of the Galactic globular clusters (GGCs) in Schiavon et al. (2005; hereafter S05) exhibit a large, intrinsic scatter that cannot be explained by their errors, particularly at intermediate-to-high metallicities. Trying to understand the nature of such scatter motivated us to carry out the present work. Further details can also be found in Cenarro et al. (2008; hereafter C08). 

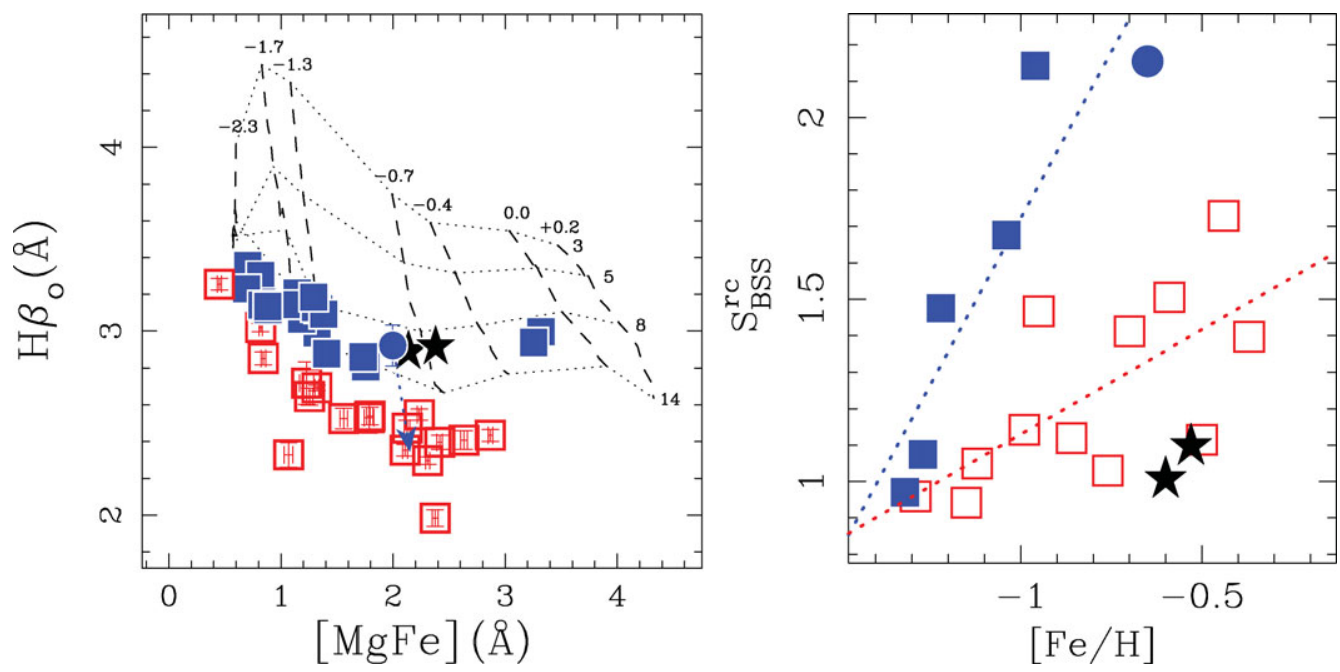

Figure 1. Left panel: $\mathrm{H} \beta_{\mathrm{o}}$ vs [MgFe] plot for the 41 GGC spectra of $\mathrm{S} 05$. Filled symbols and open red squares represent, repectively, GGCs with high (GGCHs) and low (GGCLs) H $\beta$ strengths at fixed metallicity. The SSP model grid of Vazdekis et al. (2009) is overplotted to guide the eye (ages and metallicities given in the labels). Black filled stars are the second parameter clusters NGC6388 and NGC6441. The blue filled circle is NGC6342. The arrow indicates the location of NGC6342 after correcting its spectrum for the additional contribution of BSSs (see text in Section 3 for details). Right panel: GGC $[\mathrm{Fe} / \mathrm{H}]$ vs the logarithmic specific frequency of BSSs inside the core radius, $S_{\mathrm{BSS}}^{r_{\mathrm{c}}}$, for GGCs with $[\mathrm{Fe} / \mathrm{H}]>-1.35$. Symbols as in left panel. Blue and red dotted lines illustrate linear fits to filled blue symbols and open red squares respectively.

\section{The GGC data: Integrated spectra vs CMD-based parameters}

Figure 1 (left) illustrates the $\mathrm{H} \beta_{\mathrm{o}}$ vs $[\mathrm{MgFe}]$ plot for the GGC catalogue of $\mathrm{S} 05$, a set of 41 GGC spectra integrated inside the core radius, $r_{\mathrm{c}}$. SSP models by Vazdekis et al. (2009), based on MILES (Sánchez-Blázquez et al. 2006a; Cenarro et al. 2007a), are overplotted to guide the eye, as no parameters are directly inferred from the model grid. The large scatter in the GGC $\mathrm{H} \beta_{\mathrm{o}}$ is particularly apparent at the intermediate-to-high metallicity regime $([\mathrm{MgFe}] \gtrsim 1.2 ;[\mathrm{Fe} / \mathrm{H}]>-1.35)$. A low-order polynomial fit to all data splits the GGC dataset into two subsamples: GGCs with high (GGCHs; filled symbols) and low (GGCLs; open red squares) $\mathrm{H} \beta_{\mathrm{o}}$ values at fixed [MgFe]. To facilitate further discussions, filled black stars illustrate the metal-rich (MR) second parameter clusters NGC6388 and NGC6441 (Rich et al. 1997). In addition, NGC6342, the case study of Section 3, is presented as a filled blue circle. As it can be seen in $\mathrm{C} 08, \mathrm{H} \beta$ differences between GGCHs and GGCLs are also apparent for the classical $\mathrm{H} \beta$ Lick index, although its greater age-metallicity degeneracy makes them more difficult to detect a priori.

It is therefore clear that some property differs between GGCHs and GGC $L$ s leading to different Balmer-line strengths at a given metallicity. In fact, since the $\mathrm{H} \beta_{\mathrm{o}}$ scatter looks to increase with the increasing metallicity, we focus on those 25 GGCs with $[\mathrm{Fe} / \mathrm{H}]$ $>-1.35$ among which striking differences in the $\mathrm{H} \beta$ strength at fixed metallicity are observed. For these GGCs, it has been proven that the observed differences cannot be explained by either different MSTO ages, blue HB stars, $[\alpha / \mathrm{Fe}]$ ratios, and/or Balmer lines filled-in by foreground emission (see details in C08). Additionally, Fig. 1 (right) presents the GGC metallicity (as taken from the Harris 1996 catalogue, Feb 2003 revision; hereafter H03) vs the specific frequency of BSSs inside the GGC core radius, $S_{\mathrm{BSS}}^{r_{\mathrm{c}}}$. The last quantity, computed by Moretti et al. (2008; hereafter M08) on the basis of the GGC HST/WFPC2 snapshot catalogue of Piotto et al. (2002), measures the logarithmic 
number of BSSs inside $r_{\mathrm{c}}$, normalised to the sampled luminosity -in units of $10^{4} L_{\odot}$ - in the $F 555 W$ HST band within the same aperture. Note that this CMD-derived parameter is representative of the spectroscopic data in Fig. 1 (left) as they both are measured inside $r_{\mathrm{c}}$. Interestingly, GGCHs and GGC $L \mathrm{~s}$-which were identified spectroscopically- are clearly segregated in terms of their BSS specific frequencies. At a given [Fe/H], GGCs with higher $S_{\mathrm{BSS}}^{r_{\mathrm{c}}}$ values exhibit stronger $\mathrm{H} \beta$ lines, suggesting that BSSs are responsible for their larger $\mathrm{H} \beta$ values. The only exceptions are the second parameter GGCHs NGC6388 and NGC6441 (filled black stars with low $S_{\mathrm{BSS}}^{r_{\mathrm{c}}}$ values), whose large $\mathrm{H} \beta$ strengths are known to be driven by bluer HBs. Also, we find clear correlations between $S_{\mathrm{BSS}}^{r_{\mathrm{c}}}$ and metallicity for GGCHs and GGCLs separately (dotted lines in Fig. 1 (right)), indicating that the relative contributions of BSSs can become larger at high metallicities. Note that the expected fading with metallicity in $F 555 \mathrm{~W}$ band for the most MR GGCs only accounts for up to $\Delta S_{\mathrm{BSS}}^{r_{\mathrm{c}}} \sim 0.2$, so the above trends are irrespective of this effect.

\section{Modeling the BSS population of NGC6342}

To confirm whether BSSs can indeed explain the differences in $\mathrm{H} \beta$ between GGCHs and GGCLs, we compute quantitatively the impact of the BSS population of NGC6342 -the GGCH with the highest $S_{\mathrm{BSS}}^{r_{c}}$ in our dataset (filled blue circle)- on its integrated spectrum. How does the integrated $\mathrm{H} \beta_{\mathrm{o}}$ of NGC6342 change if we subtract its BSSs?

Combining photometric data from H03 and M08 for both NGC6342 and its BSS population, we determine that $13 \%$ of the GGC flux in $V$ band within $r_{\mathrm{c}}$ comes from a population of $7 \mathrm{BSSs}$ with $B-V$ varying from 0.22 to 0.52 . For each BSS, assuming its $B-V$ and $[\mathrm{Fe} / \mathrm{H}]=-0.65$, we estimate $T_{\text {eff }}$ using the $(B-V)-T_{\text {eff }}$ relation for dwarfs from Alonso et al. (1996). The $T_{\text {eff }}$ values of the $7 \mathrm{BSSs}$ are in the range $5925 \leqslant T_{\text {eff }} \leqslant 7500 \mathrm{~K}$. We then compute spectral BSS templates with the above parameters on the basis of MILES, using the interpolating algorithm in Vazdekis et al. (2003; Appendix B). After the 7 BSS templates are scaled according to their $V$ fluxes and subtracted from the NGC6342 spectrum, its integrated $\mathrm{H} \beta_{0}$ decreases by $0.70 \AA$. However, taking into account that the averaged $S_{\mathrm{BSS}}^{r_{\mathrm{c}}}$ of $\mathrm{GGCLs}$ with $[\mathrm{Fe} / \mathrm{H}]>-0.8$ (similar to that of NGC6342) is not null but $\sim 1.4$ (Fig. 1 (right)), and assuming similar $T_{\text {eff }}$ values for their BSS populations, the relative offsets in $\mathrm{H} \beta_{\mathrm{o}}$ and [MgFe] between NGC6342 and the above GGCLs are $0.58 \AA$ and $-0.15 \AA$ respectively. Such index offsets, illustrated by the arrow in Fig. 1 (left), agree perfectly with the observed differences between NGC6342 and similar metallicity GGCLs, hence confirming quantitatively that BSSs do account for the observed differences in $\mathrm{H} \beta$ between GGCHs and GGCLs at the intermediate-to-high metallicity regime.

\section{Implications and discussion}

The present study reveals that (a) BSSs are primarily responsible for the $\mathrm{H} \beta$ variations observed among the integrated spectra of intermediate-to-high metallicity GGCs, and (b) their relative importance seems to increase with metallicity. These results may impose severe constraints to the information derived from Balmer-line based age-metallicity diagnostics of unresolved stellar populations:

i) Extragalactic globular clusters (EGCs). Since the specific frequency of BSS in EGCs is unknown, our results set a fundamental limit to the reliability with which ages can be determined for EGCs using Balmer lines and SSP models. Taking the S05 data as representative of old GC systems, and assuming that GGCLs are $\sim 14 \mathrm{Gyr}$ old, the $\mathrm{H} \beta_{\mathrm{o}}$ offsets between GGCHs and GGCLs can be misinterpreted on the basis of SSP models 
as a rejuvenation of up to $\sim 8 \mathrm{Gyr}$. Closely related to this, the MR subpopulation of EGCs is sometimes found to look younger -on average- with a larger age scatter than the metal-poor (MP) one (e.g. Puzia et al. 2005). Such behaviour clearly resembles the picture in Fig 1 (left). Albeit our current results do not rule out the existence of true age differences between MP and MR EGC subpopulations, the increasing importance of BSSs with metallicity might, at least, partially drive the younger ages reported by some previous work.

ii) Galaxies. Can BSSs have an important effect on the integrated ages of galaxies? The answer to this question certainly depends on the nature of BSSs. Stellar collisions driving BSSs (e.g. Davies et al. 1994) are expected to be highly inefficient in galaxies because of their low stellar densities. However, BSSs generated via mass-transfer binaries (McCrea 1964) could be a non-negligible phenomenom, as it has been reported by e.g. Momany et al. (2007) and Mapelli et al. (2007) for dwarf spheroidal galaxies, which possess even larger $S_{\mathrm{BSS}}^{r_{\mathrm{c}}}$ values than GGCs. In addition, if the increasing importance of BSSs with metallicity observed in Fig. 1 (right) holds for the higher metallicities of massive ellipticals, it is immediate to wonder whether different BSS ratios may be driving partially the scatter among the luminosity-weighted $\mathrm{H} \beta$ strengths of large spheroids reported in the literature. Further work in this direction is however needed to shed light on the current issue.

\section{References}

Alonso, A., Arribas, S., \& Martínez-Roger, C. 1996, A\&A, 313, 873

Cenarro, A. J., Peletier, R. F., Sńchez-Blzquez, P., Selam, S. O., Toloba, E., Cardiel, N., FalcónBarroso, J., Gorgas, J., Jiménez-Vicente, J., \& Vazdekis, A. 2007a, MNRAS, 374, 664

Cenarro, A. J., Beasley, M. A., Strader, J., Brodie, J. P., \& Forbes, D. A. 2007b, AJ, 134, 391

Cenarro, A. J., Cervantes, J.L., Beasley, M. A., Marin-Franch, A., \& Vazdekis A., 2008, ApJ, $689 \mathrm{~L}, 29$

Cervantes, J. L. \& Vazdekis, A. 2009, MNRAS, 391, 691

Davies, M. B., Benz, W., \& Hills, J. G. 1994, ApJ, 424, 870

Harris, W. E. 1996, AJ, 112, 1487

Lee, H., Yoon, S., \& Lee, Y. 2000, AJ, 120, 998

Mapelli, M., Ripamonti, E., Tolstoy, E., Sigurdsson, S., Irwin, M. J., \& Battaglia, G. 2007, MNRAS, 380, 1127

McCrea, W. H. 1964, MNRAS, 128, 147

Momany, Y., Held, E. V., Saviane, I., Zaggia, S., Rizzi, L., \& Gullieuszik, M. 2007, A\&A, 468, 973

Moretti, A., De Angeli, F., \& Piotto, G. 2008, A\&\&A, 483, 183 (M08)

Piotto et al. 2002, A\& A, 391, 945

Puzia, T. H., Kissler-Patig, M., Thomas, D., Maraston, C., Saglia, R. P., Bender, R., Goudfrooij, P., \& Hempel, M., 2005, A\&A A, 439, 997

Rose, J. A. 1985, AJ, 90, 1927

Sánchez-Blázquez, P., Peletier, R. F., Jiménez-Vicente, J., Cardiel, N., Cenarro, A. J., FalcónBarroso, J., Gorgas, J., Selam, S., \& Vazdekis, A. 2006, MNRAS, 371, 703

Schiavon, R. P., Rose, J. A., Courteau, S., \& MacArthur, L. A. 2004, ApJ, 608, 33

Schiavon, R. P., Rose, J. A., Courteau, S., \& MacArthur, L. A. 2005, ApJS, 160, 163 (S05)

Trager, S. C., Worthey, G., Faber, S. M., \& Dressler, A. 2005, MNRAS, 362, 2

Vazdekis, A., Salaris, M., Arimoto, N., \& Rose, J. A. 2001, ApJ, 549, 274

Vazdekis, A., Sánchez-Blázquez, P., Falcón-Barroso, J., Cenarro, A. J., Beasley, M. A., Cardiel, N., Gorgas, J., \& Peletier, R., 2009, MNRAS, submitted, arXiv:XXXX

Vazdekis, A., Cenarro, A. J., Gorgas, J., Cardiel, N., \& Peletier, R. F. 2003, MNRAS, 340, 1317 